\title{
EXTENSÃO DO MODELO DE MISME E FIMBEL PARA A DETERMINAÇÃO DA DISTRIBUIÇÃO CUMULATIVA DA ATENUAÇÃO DIFERENCIAL DEVIDA À ÇHUVA ENTRE DOIS ENLACES CONVERGENTES
}

\author{
Henrique Grynszpan e Emanoel Costa
}

\begin{abstract}
Resumo - Apresenta-se um modelo não paramétrico para a distribuição cumulativa da atenuação diferencial devida à chuva entre dois enlaces convergentes operando em freqüências superiores a 10 GHz. Estende-se aqui o modelo de Misme e Fimbel, que determina a distribuição cumulativa da atenuação devida à chuva em um enlace isolado, a partir de seus parâmetros e de dados da região radioclimática, assim como de conceitos da teoria das probabilidades. Em seguida, comparam-se previsões do modelo e resultados experimentais e estudam-se os efeitos das variações do ângulo entre os enlaces e dos seus comprimentos, assim como os efeitos da chuva sobre a distribuição cumulativa da relação $\mathrm{C} / \mathrm{I}$, considerando a discriminação angular da antena receptora.
\end{abstract}

Palavras-Chave - Atenuação diferencial devida à chuva, Enlaces convergentes, Interferências entre enlaces rádio terrestres.

Abstract - A non-parametric model is presented for the cumulative distribution of the differential rain attenuation between two converging links operating at frequencies above $10 \mathrm{GHz}$. This is accomplished through an extension of the Misme and Fimbel model, which determines the cumulative distribution of the rain attenuation in an isolated link from its parameters and data from the radioclimatic region, as well as from concepts of the probability theory. Next, model predictions and experimental results are compared and effects from variations of the angle between the links and of the path length, as well as from rain on the cumulative distribution of the $\mathrm{C} / \mathrm{I}$ ratio, considering the angular discrimination of the receiving antenna, are studied.

Keywords - Differential rain attenuation, Converging links, Interference between terrestrial radio links.

\section{INTRODUÇÃO}

As faixas de frequiência acima de $10 \mathrm{GHz}$ têm sido muito utilizadas no acesso rádio ao cliente, numa configuração do tipo estrela, com enlaces rádio convergentes em uma estação nodal. Observou-se que o cálculo da indisponibilidade devida à chuva destes enlaces era realizado de maneira conservadora, causando uma utilização relativamente ineficiente do espectro de frequêencias. No cálculo, era considerada apenas a atenuação devida à chuva no enlace desejado, supondo que o enlace interferente não sofria atenuação. A consideração de que a célula de chuva atenua ambos os enlaces (desejado e interferente) traz como consequiência importante a possibilidade de redução do ângulo mínimo de projeto entre dois enlaces convergentes, permitindo o aumento da eficiência espectral. De fato, para aumentar a eficiência espectral, é necessário que o número de enlaces convergentes na estação nodal aumente, atendendo a um grande número de estações remotas, e que uma mesma frequiência seja reutilizada o maior número de vezes possível neste conjunto de enlaces.

Henrique Grynszpan é engenheiro da Petróleo Brasileiro S. A. (PETROBRAS) e aluno de PósGraduação do Centro de Estudos em Telecomunicações da Pontifícia Universidade Católica do Rio de Janeiro, Rio de Janeiro, Brasil (CETUC/PUC-Rio). Emanoel Costa é professor do CETUC/PUCRio. E-mails: henriquegrynszpan@petrobras.com.br, epoc@cetuc.puc-rio.br.
Diversos métodos foram propostos na literatura para a determinação da distribuição cumulativa da atenuação diferencial entre enlaces convergentes. Morita e colaboradores [1],[2] propuseram a distribuição Gama para a função densidade de probabilidade conjunta das atenuações nos dois enlaces, supostas correlacionadas. Os parâmetros desta função foram determinados a partir da distribuição cumulativa probabilidade da taxa de precipitação medida e das características espaciais da estrutura da precipitação. Em seguida, a função distribuição cumulativa da atenuação diferencial entre os dois enlaces foi determinada numericamente. As previsões do modelo foram comparadas com os resultados de medidas realizadas no Electrical Communication Laboratory (ECL), Japão. Os trabalhos de Kanellopoulus e colaboradores [3],[4] são análogos aos de Morita e colaboradores [1],[2], exceto pela suposição de distribuições Lognormais para as distribuições cumulativas da taxa de precipitação e da atenuação em um enlace, assim como para a densidade de probabilidade conjunta das atenuações nos dois enlaces. As previsões do modelo foram comparadas com os resultados de medidas realizadas pelo CETUC/PUC-Rio em lances operados pela Embratel e disponíveis na literatura [5]. Recentemente, Paulson et al. [6] também adotaram o modelo Lognormal para a densidade de probabilidade conjunta das atenuações em dois enlaces. Entretanto, os parâmetros da distribuição foram estimados a partir de medidas realizadas pelo radar meteorológico de Chibolton, Reino Unido. Em seguida, o modelo foi utilizado para estimar o fator de melhoria de diversidade (DI) e o ganho de diversidade (DG) em enlaces convergentes. Por outro lado, o estudo de Garcia et al. [7] baseou-se em medidas da distribuição da atenuação diferencial em diversos pares de enlaces, bem como nas medidas da distribuição de atenuação por chuva de cada enlace. Tais medidas foram realizadas na estação Rua dos Ingleses, São Paulo bem como em Brasília. A predição baseia-se na seguinte expressão analítica, cujos coeficientes resultaram de ajuste aos dados experimentais

$$
\mathrm{A}_{\mathrm{AB}}=\left(\mathrm{A}_{\mathrm{A}}-0.34 \mathrm{~A}_{\mathrm{B}}\right) \cdot\left(2.65|\theta|^{0.23}+0.004|\Delta \mathrm{d}|^{2.25}\right) \cdot \mathrm{f}^{-0.4}
$$

Nesta expressão, ? (rad) é o ângulo entre os enlaces, ?d $(\mathrm{km})$ é a diferença entre os comprimentos dos enlaces, $\mathrm{f}$ $(\mathrm{GHz})$ é a freqüência de operação, $\mathrm{A}_{\mathrm{AB}}$ é a atenuação diferencial e $A_{A}$ e $A_{B}$ são as atenuações individuais dos enlaces, todas excedidas durante a mesma percentagem $\mathrm{p} \%$ do tempo.

Este trabalho apresenta um modelo não paramétrico para a determinação da distribuição cumulativa da atenuação 
diferencial devida à chuva entre dois enlaces convergentes operando em freqüências superiores a $10 \mathrm{GHz}$. O desenvolvimento estende o modelo de Misme e Fimbel [8], que determina a distribuição cumulativa da atenuação devida à chuva em um enlace isolado a partir de seus parâmetros e de dados radiometeorológicos da região, assim como de conceitos da teoria das probabilidades. O modelo a ser descrito determina, inicialmente, a distribuição cumulativa conjunta das atenuações devidas à chuva nos dois enlaces. A partir da distribuição cumulativa conjunta, obtém-se a distribuição da atenuação diferencial entre os dois enlaces. Este modelo generaliza o trabalho apresentado por Stola [9], limitado à determinação da distribuição cumulativa da atenuação simultaneamente excedida em dois enlaces convergentes, também a partir dos procedimentos propostos por Misme e Fimbel.

\section{O MODELO DE MISME E FIMBEL ORIGINAL E SUA EXTENSÃO}

O modelo de Misme e Fimbel [8], com as pequenas modificações introduzidas aqui, adota as seguintes características para a precipitação devida à chuva: (1) a precipitação intensa se apresenta sob a forma de células de chuva cilíndricas circulares verticais de taxa de precipitação $\mathrm{R}(\mathrm{mm} / \mathrm{h})$ constante; (2) o diâmetro $\mathrm{d}(\mathrm{km})$ de uma célula de chuva se relaciona à taxa de precipitação no seu interior por intermédio da relação

$$
d(R)=d_{o}(100 / R)^{\beta}
$$

(3) a taxa de precipitação em um ponto arbitrário da região de interesse varia aleatoriamente no tempo, sendo caracterizada pela sua distribuição cumulativa $\mathrm{P}_{\mathrm{r}}(\mathrm{R})$, que independe do ponto de observação; (4) em cada instante de tempo, a atenuação em um enlace é causada por apenas uma célula de chuva; (5) a atenuação específica $\gamma(\mathrm{dB} / \mathrm{km})$ se relaciona à taxa de precipitação por intermédio da relação $\gamma=\mathrm{k} \mathrm{R}^{\alpha}$, sendo os parâmetros $\mathrm{k}$ e $\alpha$ determinados em função da freqüência de operação e da polarização de acordo com a versão mais recente da Recomendação ITU-R P.838 [10]. Considerando que a contribuição da precipitação residual especificada originalmente [8] para a atenuação é pequena, seus efeitos foram desprezados aqui.

A interseção entre uma célula de taxa de precipitação R e o enlace $\mathrm{AB}$ observado na Figura 1, cujo comprimento é $\mathrm{D}_{1}$ $(\mathrm{km})$, é uma corda de comprimento $\mathrm{L}_{\mathrm{o}}(\mathrm{km})$, que provoca no enlace a atenuação $A_{o}(d B)$ igual a

$$
\mathrm{A}_{\mathrm{o}}=\mathrm{kR}^{\alpha} \mathrm{L}_{\mathrm{o}} \rightarrow \mathrm{L}_{\mathrm{o}}\left(\mathrm{A}_{\mathrm{o}}, \mathrm{R}\right)=\mathrm{A}_{\mathrm{o}} /\left(\mathrm{kR}^{\alpha}\right)
$$

Para cada valor de $A_{0}$, existe uma taxa de precipitação mínima $R_{\min }\left(A_{o}\right)$ que garante que, simultaneamente, $L_{o}\left(A_{o}\right.$, $\left.R_{\text {min }}\right) \leq d\left(R_{\min }\right)$ e $L_{o}\left(A_{o}, R_{\min }\right) \leq D$. Este valor deve ser determinado a partir das igualdades correspondentes. Em seguida, com base no Teorema da Probabilidade Total [11] e em conceitos geométricos, apresenta a seguinte equação geral para a distribuição cumulativa da atenuação devida à chuva no enlace $\mathrm{AB}$ isolado

$$
\operatorname{Pr}\left\{\mathrm{A}>\mathrm{A}_{\mathrm{o}}\right\}=\frac{4}{\pi} \int_{\mathrm{P}_{\min }}^{1} \frac{\mathrm{S}\left[\mathrm{A}_{\mathrm{o}}, \mathrm{R}\left(\mathrm{P}_{\mathrm{r}}\right)\right]}{\mathrm{d}^{2}\left[\mathrm{R}\left(\mathrm{P}_{\mathrm{r}}\right)\right]} \mathrm{dP}_{\mathrm{r}}
$$

Nesta equação, $\mathrm{S}\left(\mathrm{A}_{\mathrm{o}}, \mathrm{R}\right)$ é a área do lugar geométrico associado ao enlace $A B$, tal que uma célula de taxa de precipitação $R$, diâmetro $d(R)$ e de centro situado no seu interior provoca atenuação superior a $A_{o}(d B)$. $O$ contorno do lugar geométrico é apresentado por linhas tracejadas na Figura 1 e sua área é igual a

$$
\begin{gathered}
S\left(A_{o}, R\right)=\left(D_{1}-L_{o}\right) \sqrt{d^{2}-L_{o}^{2}}+ \\
+\frac{1}{2}\left[d^{2} \arctan \left(\frac{\sqrt{d^{2}-L_{o}^{2}}}{L_{o}}\right)-L_{o} \sqrt{d^{2}-L_{o}^{2}}\right]
\end{gathered}
$$

Como ferramenta auxiliar à extensão do modelo descrito acima, a Figura 1 representa dois enlaces convergentes de comprimentos $D_{1}$ e $D_{2}$ que formam o ângulo $\theta$ na estação comum, juntamente com os lugares geométricos associados a uma célula de taxa de precipitação $R$ e diâmetro $d(R)$, assim como às atenuações $A_{o 1}$ e $A_{02}$, respectivamente.

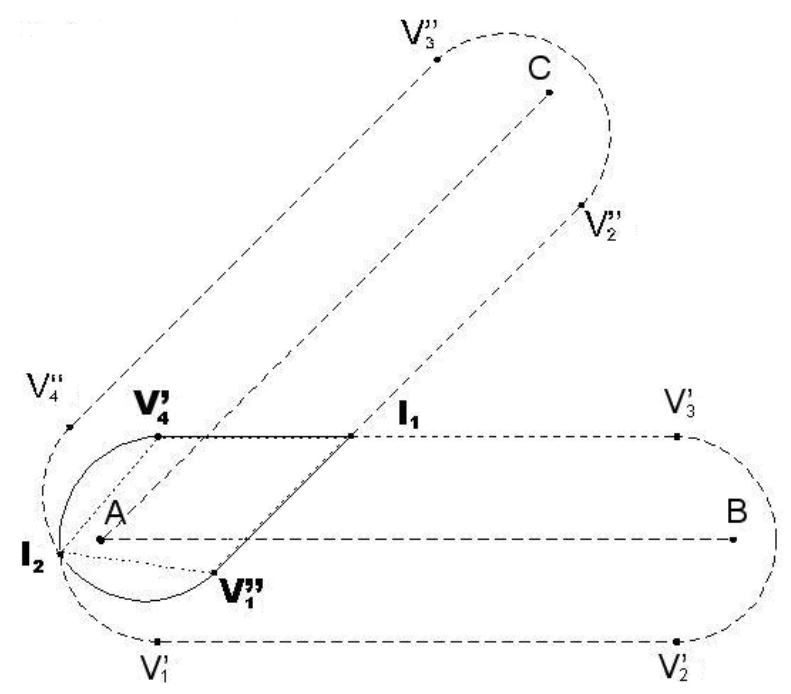

Figura 1. Enlaces convergentes $\mathrm{AB}$ e $\mathrm{AC}$ de comprimentos $\mathrm{D}_{1}$ e $\mathrm{D}_{2}$ que formam o ângulo $\theta$ na estação comum, juntamente com os lugares geométricos associados a uma célula de taxa de precipitação $\mathrm{R}$ e diâmetro $\mathrm{d}(\mathrm{R})$ e às atenuações $\mathrm{A}_{01}$ e $\mathrm{A}_{02}$, respectivamente, assim como interseção $\mathrm{I}_{2} \mathrm{~V}_{1} \mathrm{I}_{1} \mathrm{~V}_{4}{ }_{4}$ entre os dois lugares geométricos

Com base no desenvolvimento apresentado nos parágrafos anteriores, a probabilidade conjunta de que, simultaneamente, a atenuação $A_{1}$ no primeiro enlace exceda $A_{o 1}$ e a atenuação $A_{2}$ no segundo enlace exceda $A_{o 2}$ pode 
ser imediatamente representada por intermédio da expressão

$$
\operatorname{Pr}\left\{\mathrm{A}_{1}>\mathrm{A}_{\mathrm{o} 1}, \mathrm{~A}_{2}>\mathrm{A}_{\mathrm{o} 2}\right\}=\frac{4}{\pi} \int_{\mathrm{P}_{\min }}^{1} \frac{\mathrm{S}_{\bigcap}\left[\mathrm{A}_{\mathrm{o} 1}, \mathrm{~A}_{\mathrm{o} 2}, \mathrm{R}\left(\mathrm{P}_{\mathrm{r}}\right)\right]}{\mathrm{d}^{2}\left[\mathrm{R}\left(\mathrm{P}_{\mathrm{r}}\right)\right]} \mathrm{dP}_{\mathrm{r}}(6)
$$

Nesta expressão, $\mathrm{S}_{\mathrm{n}}\left(\mathrm{A}_{\mathrm{o} 1}, \mathrm{~A}_{\mathrm{o} 2}, \mathrm{R}\right)$ é a interseção entre as áreas limitadas pelos dois lugares geométricos e $\mathrm{P}_{\min } \mathrm{O}$ valor de $P_{r}\left(R_{\min }\right)$, onde $R_{\min }=\max \left\{R_{\min 1}, R_{\min 2}\right\}$, sendo $R_{\min 1,2}$ a taxa de precipitação mínima para cada enlace. A equação (6) estende aquela apresentada por Stola [9], que analisou apenas o caso particular $A_{o 1}=A_{02}$. Especificada $a$ configuração de interesse, as integrais apresentadas na expressão (6) são calculadas numericamente para cada par de valores de atenuações $\left(\mathrm{A}_{\mathrm{o} 1}, \mathrm{~A}_{\mathrm{o} 2}\right)$ definidos sobre grade uniforme, sendo a área $\mathrm{S}_{\mathrm{n}}\left(\mathrm{A}_{\mathrm{o} 1}, \mathrm{~A}_{\mathrm{o} 2}, \mathrm{R}\right)$ determinada exatamente por intermédio do algoritmo descrito a seguir. Observa-se que os lugares geométricos são convexos, de modo que a interseção entre eles também será.

Os vértices que determinam a interseção são de dois tipos: (1) ponto interior, existente quando um vértice de um lugar geométrico está no interior do outro lugar geométrico; e (2) pontos de interseção, existente quando segmentos de lugares geométricos distintos se cortam. Todos estes pontos são determinados por intermédio de algoritmos apropriados de geometria computacional [12] e, em conjunto, definem os vértices da interseção entre os dois lugares geométricos. Em seguida, estes pontos são ordenados no sentido antihorário, determinando-se também se segmentos formados por vértices consecutivos devem ser conectados por segmento de reta ou arco de circunferência e, no segundo caso, a posição do centro do arco. Finalmente, o polígono é decomposto em triângulos formados pelos seus lados e pelas diagonais traçadas a partir de qualquer de seus vértices e por segmentos circulares. $A$ área $S_{n}\left(A_{o 1}, A_{o 2}, R\right)$ comum aos dois lugares geométricos é exatamente igual à soma das áreas destes elementos.

A distribuição cumulativa complementar da atenuação diferencial pode ser determinada diretamente a partir dos resultados da expressão (6) por intermédio da aproximação

$$
\begin{gathered}
\mathrm{C}_{\mathrm{a}_{1}-\mathrm{a}_{2}}\left(\mathrm{~A}_{\mathrm{o}}\right)=\operatorname{Pr}\left\{\mathrm{a}_{1}-\mathrm{a}_{2}>\mathrm{A}_{\mathrm{o}}\right\} \approx \\
\approx \sum_{\mathrm{i}=1}^{\mathrm{N}} \operatorname{Pr}\left\{\mathrm{a}_{1}>\mathrm{A}_{2 \mathrm{i}}+\mathrm{A}_{\mathrm{o}},>\mathrm{A}_{2 \mathrm{i}}-\varepsilon / 2<\mathrm{a}_{2}<\mathrm{A}_{2 \mathrm{i}}+\varepsilon / 2\right\} \\
\approx \sum_{\mathrm{i}=1}^{\mathrm{N}}\left[\operatorname{Pr}\left\{\mathrm{a}_{1}>\mathrm{A}_{2 \mathrm{i}}+\mathrm{A}_{\mathrm{o}}, \mathrm{a}_{2}>\mathrm{A}_{2 \mathrm{i}}-\varepsilon / 2\right\}-\right. \\
\left.-\operatorname{Pr}\left\{\mathrm{a}_{1}>\mathrm{A}_{2 \mathrm{i}}+\mathrm{A}_{\mathrm{o}}, \mathrm{a}_{2}>\mathrm{A}_{2 \mathrm{i}}+\varepsilon / 2\right\}\right]
\end{gathered}
$$

onde $\varepsilon$ representa a resolução da grade uniforme. Portanto, o somatório é realizada sobre regiões retangulares de largura compreendida entre $A_{1 i}=\left(A_{2 i}+A_{o}\right)$ e $A_{1 \max }$ e de alturas $\varepsilon$ centradas em $\mathrm{A}_{2 \mathrm{i}}$. Testes realizados comprovaram que este somatório fornece uma excelente aproximação quando o valor de $\mathrm{A}_{\mathrm{o}}$ excede alguns decibéis.

\section{PREVISÕES DO MODELO E COMPARAÇÕES COM MEDIDAS}

Para estender a aplicabilidade do modelo de Misme e Fimbel aos enlaces apresentados nas Figuras 2 e 3, os valores originais dos parâmetros da expressão (2) foram substituídos por $d_{o}=7 \mathrm{~km}$ e $\beta=0,4$ para comparações entre previsões de modelos e medidas realizadas em São Paulo e $\mathrm{d}_{\mathrm{o}}=2,8 \mathrm{~km}$ e $\beta=0,14$ para comparações entre previsões de modelos e medidas realizadas no Japão. Nas faixas de frequiências utilizadas, têm-se, para a polarização vertical de todos os enlaces utilizados, $\mathrm{a}_{\mathrm{V}}=1,128$ e $\mathrm{k}_{\mathrm{V}}=0,03350$ para $\mathrm{f}$ $=15 \mathrm{GHz}$ e $\mathrm{a}_{\mathrm{V}}=1,1088$ e $\mathrm{k}_{\mathrm{V}}=0,0661$ para $\mathrm{f}=19 \mathrm{GHz}$.

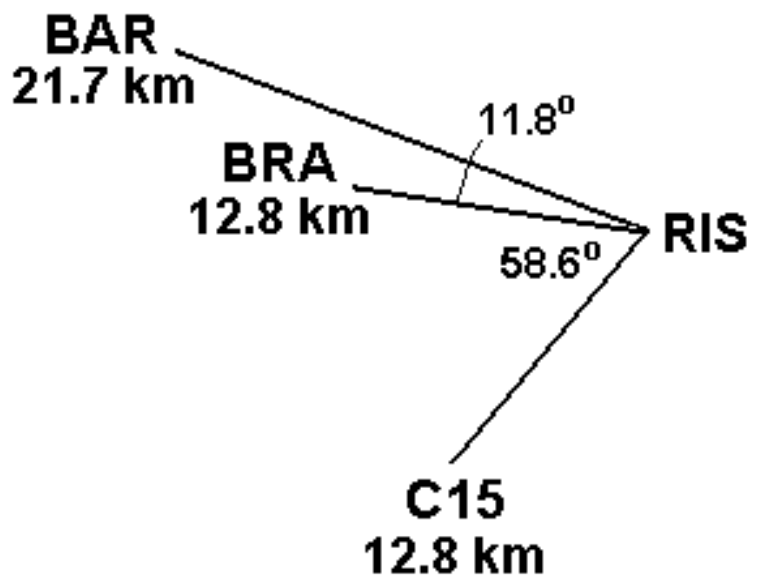

Figura 2. Posições relativas dos enlaces convergentes na estação de Rua dos Ingleses (RIS), São Paulo, utilizados nos testes do modelo de previsão

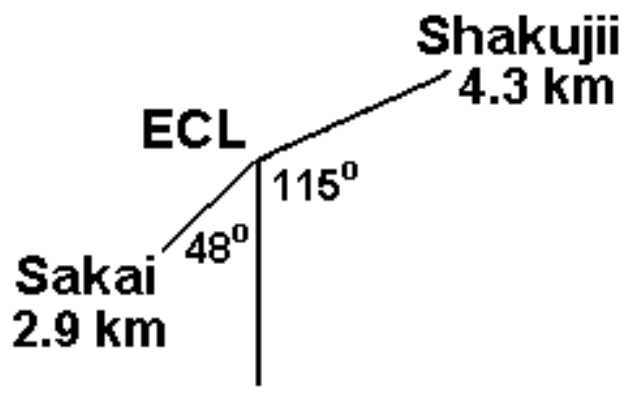

$\underset{4.7 \mathrm{~km}}{\text { Shinkawa }}$

Figura 3. Posições relativas dos enlaces convergentes no Masashino Electrical Communication Laboratory (ECL), Japão, utilizados nos testes do modelo de previsão (adaptada de [2])

Inicialmente, esta seção compara previsões do modelo com os resultados experimentais correspondentes, obtidos pelo CETUC/PUC-Rio em enlaces convergentes da Embratel situados na cidade de São Paulo [5],[7] e pelo Electrical Communication Laboratory no Japão [2]. A distribuição cumulativa complementar da atenuação diferencial estão apresentadas nas Figuras 4 a 7.

A distribuição cumulativa da atenuação diferencial entre enlaces de mesmo comprimento deveriam ser simétricas, 
como indicam as curvas teóricas BRA-C15 e C15-BRA da Figura 4. Para a mesma percentagem de tempo, enlaces mais longos deveriam apresentar maiores atenuações diferenciais, como indicam as curvas teóricas C15-BAR e BAR-C15 da Figura 5. Entretanto, a Figura 4 apresenta diferenças de até $5 \mathrm{~dB}$ entre as distribuições cumulativas medidas BRA-C15 e C15-BRA, apesar de os enlaces envolvidos terem o mesmo comprimento. Por outro lado, as diferenças observadas na Figura 5 entre as distribuições cumulativas medidas C15-BAR e BAR-C15, inferiores a 4 $\mathrm{dB}$, não refletem a considerável diferença entre os comprimentos dos dois enlaces. Estas observações são creditadas à existência de direção predominante dos ventos na região, alinhada com o enlace C15-RIS, que aumenta a percentagem de tempo em que sua atenuação devida à chuva é excedida, o que explicaria as maiores diferenças observadas entre as previsões do modelo e as medidas correspondentes às configurações C15-BRA e C15-BAR.

As atenuações diferenciais medidas apresentadas nas Figuras 4 e 5 tendem a valores constantes para percentagens de tempo inferiores a $0,01 \%$. Isto é decorrente da limitação

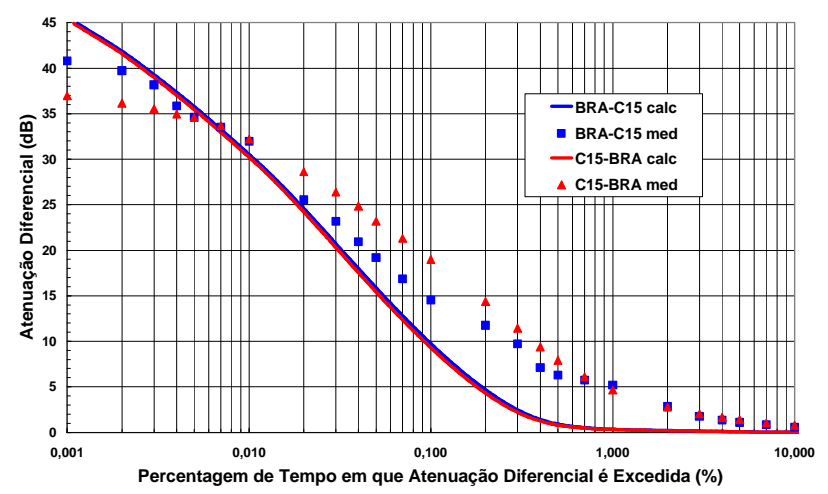

Figura 4. Atenuações diferenciais calculadas (curvas contínuas) e medidas (símbolos) entre os enlaces Bradesco II - Rua dos Ingleses $(12,8 \mathrm{~km}) \mathrm{e}$ Cenesp 15 - Rua dos Ingleses (12,8 km), que formam o ângulo de 58,6 operando na freqüência de $15 \mathrm{GHz}$

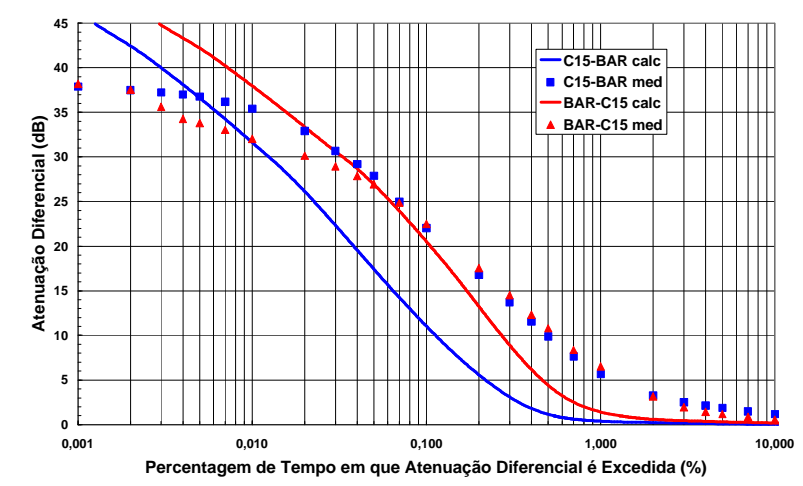

Figura 5. Atenuações diferenciais calculadas (curvas contínuas) e medidas (símbolos) entre os enlaces Cenesp 15 - Rua dos Ingleses (12,8 km) e Barueri - Rua dos Ingleses $(21,1 \mathrm{~km})$, que formam o ângulo de $70.4^{\circ}$, operando na freqüência de $15 \mathrm{GHz}$ física do receptor, que atinge o seu limiar de recepção para atenuações da ordem $30 \mathrm{~dB}$ a $40 \mathrm{~dB}$. Como fatores adicionais capazes de explicar as diferenças observadas entre as distribuições calculadas e medidas, deve-se lembrar que foi suposto que a taxa de precipitação é uniforme no interior da célula de chuva e que apenas uma célula atenua os enlaces em cada instante de tempo.

Os resultados correspondentes ao experimento realizado pelo Electrical Communication Laboratory no Japão estão apresentados nas Figuras 6 e 7.

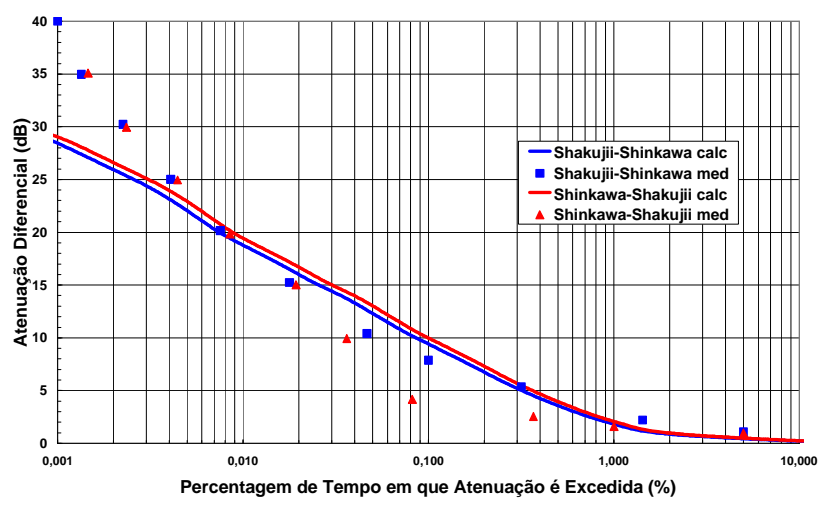

Figura 6. Atenuações diferenciais calculadas (curvas contínuas) e medidas (símbolos) entre os enlaces Shakujii - Masashino Electrical Communication Laboratory $(4,3 \mathrm{~km})$ e Shinkawa - Masashino Electrical Communication Laboratory $(4,7 \mathrm{~km})$, que formam o ângulo de $115^{\circ}$, operando na freqüência de $19 \mathrm{GHz}$ (adaptada de [2])

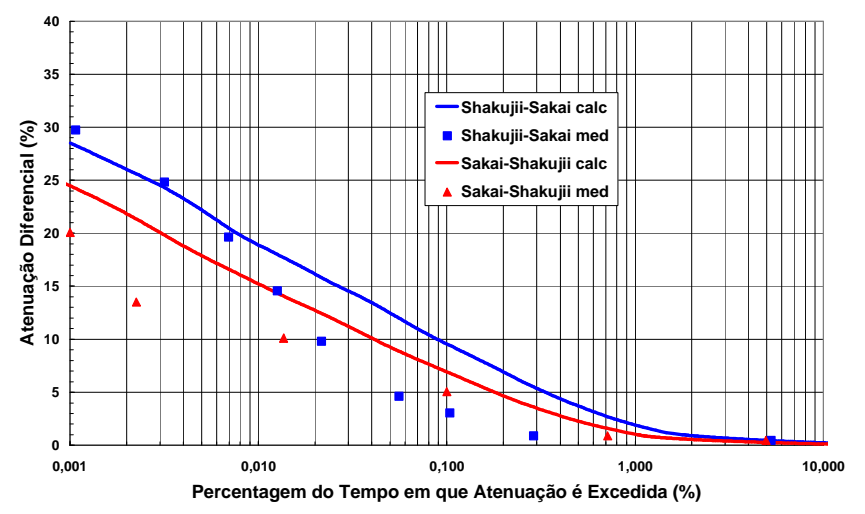

Figura 7. Atenuações diferenciais calculadas (curvas contínuas ) e medidas (símbolos) entre os enlaces Shakujii - Masashino Electrical Communication Laboratory $(4,3 \mathrm{~km})$ e Sakai - Masashino Electrical Communication Laboratory (2,9 km), que formam o ângulo de $163^{\circ}$, operando na frequiência de $19 \mathrm{GHz}$ (adaptada de [2])

Em seguida, foram determinadas as distribuições cumulativas da atenuação diferencial entre enlaces de comprimentos iguais a10 km e $20 \mathrm{~km}$ situados na região de São Paulo (isto é, supondo $d_{o}=7 \mathrm{~km} \mathrm{e} \beta=0,4$ ), para ângulos variando entre $5^{\circ}$ e $45^{\circ}$ com intervalos de $5^{\circ}$ e entre $45^{\circ}$ e $90^{\circ}$ com intervalos de $15^{\circ}$. Observou-se que, para ângulos inferiores a aproximadamente $30^{\circ}$, as curvas 
variavam rapidamente em função do incremento no ângulo entre os enlaces. Entretanto, a partir deste limiar, a mesma variação tornou-se extremamente lenta. Para evidenciar tal fato, a Figura 8 apresenta a relação entre o ângulo formado pelos enlaces e os valores da atenuação diferencial correspondentes às percentagens de tempo de iguais a $0,1 \%$ (curva tracejada) e $0,01 \%$ (curva contínua).

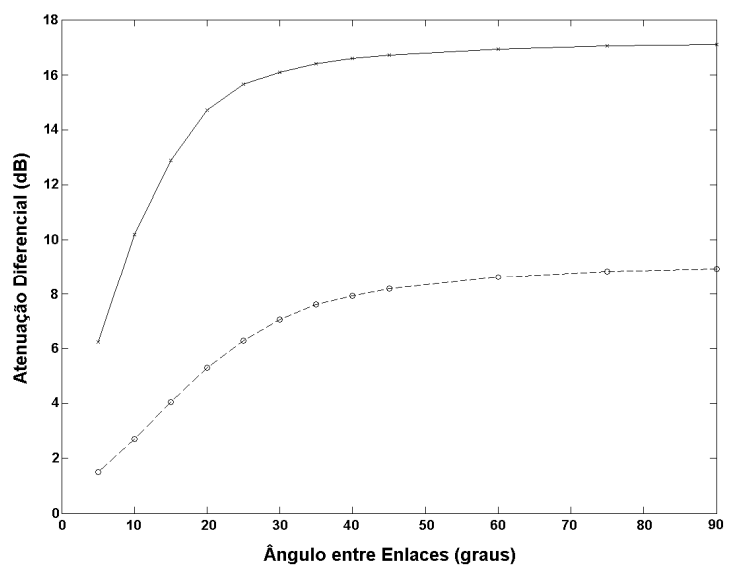

Figura 8. Relação entre o ângulo formado pelos enlaces e os valores da atenuação diferencial correspondentes às percentagens de tempo de iguais a $0,1 \%$ (curva tracejada) e $0,01 \%$ (curva contínua), para enlace de $10 \mathrm{~km}$ e $20 \mathrm{~km}$ de comprimento

Adicionalmente, o comprimento de um dos enlaces foi variado $(2 \mathrm{~km}, 3 \mathrm{~km}, 4 \mathrm{~km}, 5 \mathrm{~km}, 7 \mathrm{~km}, 10 \mathrm{~km}, 15 \mathrm{~km}, 20$ $\mathrm{km}, 25 \mathrm{~km}, 30 \mathrm{~km}, 60 \mathrm{~km}$ ), sendo fixados o comprimento do outro enlace em $10 \mathrm{~km}$ e o ângulo entre eles em $15^{\circ}$. Observou-se que, para comprimentos inferiores a $10 \mathrm{~km}$, as curvas variavam em função do incremento no comprimento do enlace. Entretanto, a partir deste limiar, esta variação não mais ocorreu. Para evidenciar tal fato, a Figura 9 apresenta a relação entre o comprimento do enlace e os valores da atenuação diferencial correspondentes às percentagens de tempo de iguais a $0,1 \%$ (curva tracejada) e $0,01 \%$ (curva contínua).

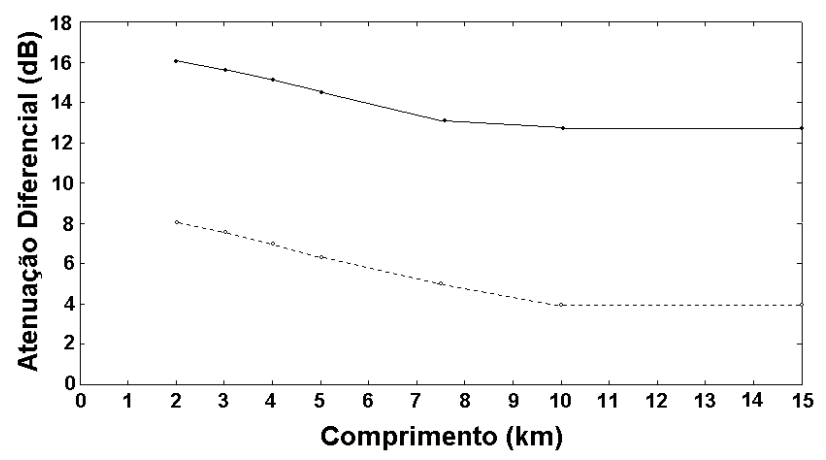

Figura 9. Relação entre o comprimento variável de um enlace e os valores da atenuação diferencial correspondentes às percentagens de tempo de iguais a $0,1 \%$ (curva tracejada) e $0,01 \%$ (curva contínua), fixados o comprimento do outro enlace em $10 \mathrm{~km}$ e o ângulo entre eles em $15^{\circ}$

\section{EFEITO DA CHUVA SOBRE A INDISPONIBILIDADE DE UM ENLACE}

Esta seção apresenta as características da relação C/I (dB) entre as potências dos sinais desejado e interferente, supondo a existência de apenas dois enlaces convergentes de $8 \mathrm{~km}$ de comprimento operando na freqüência de 15 $\mathrm{GHz}$ sob as condições climáticas da região de São Paulo. O primeiro procedimento de cálculo analisado é conservador e considera a atenuação $\mathrm{A}_{\mathrm{d}}(\mathrm{dB})$ devida à chuva no enlace desejado e despreza a atenuação $\mathrm{A}_{\mathrm{i}}(\mathrm{dB})$ no enlace interferente, de modo que a relação $\mathrm{C} / \mathrm{I}$ pode ser apresentada na forma $\mathrm{C} / \mathrm{I}=(\mathrm{C} / \mathrm{I})_{\mathrm{o}}-\mathrm{A}_{\mathrm{d}}$. O segundo procedimento é realista e considera ambas as atenuações, de modo que a relação $\mathrm{C} / \mathrm{I}$ pode ser apresentada na forma $\mathrm{C} / \mathrm{I}=$ $(\mathrm{C} / \mathrm{I})_{\mathrm{o}}-\left(\mathrm{A}_{\mathrm{d}}-\mathrm{A}_{\mathrm{i}}\right)=(\mathrm{C} / \mathrm{I})_{\mathrm{o}}-\mathrm{A}_{\text {dif. }}$ Em ambos as expressões, $(\mathrm{C} / \mathrm{I})_{0}$ representa o valor nominal da relação $\mathrm{C} / \mathrm{I}$, existente em condições de céu claro. Foram considerados os valores de $50 \mathrm{~dB}, 45 \mathrm{~dB}$ e $40 \mathrm{~dB}$ para a relação $(\mathrm{C} / \mathrm{I})_{\mathrm{o}}$, fornecidos por uma antena de alta qualidade para os ângulos de discriminação de $50^{\circ}, 20^{\circ}, 12^{\circ}$, respectivamente. Tanto $A_{d}$ quanto $A_{d i f}=A_{d}-A_{i}$ são consideradas variáveis aleatórias, cujas distribuições cumulativas foram determinadas pelos modelos descritos na seção 2, considerando os ângulos de discriminação, quando aplicável. A partir dos resultados obtidos e das expressões apresentadas acima, a distribuição cumulativa da relação $\mathrm{C} / \mathrm{I}$ foi obtida para cada procedimento e valor de $(\mathrm{C} / \mathrm{I})_{\mathrm{o}}$. A Figura 10 apresenta um conjunto de três distribuições cumulativas da relação $\mathrm{C} / \mathrm{I}$ para cada procedimento. O primeiro conjunto (à direita) corresponde ao procedimento conservador, que supõe que o enlace interferente não sofre atenuação devida à chuva. $\mathrm{O}$ segundo conjunto (à esquerda) corresponde ao procedimento realista, que considera a atenuação devida à chuva em ambos os enlaces. Os resultados indicam que, para o mesmo valor da relação $(\mathrm{C} / \mathrm{I})_{\mathrm{o}}$ (ou, equivalentemente, da separação angular entre os enlaces), a percentagem de tempo em que um determinado nível da relação $\mathrm{C} / \mathrm{I}$ não é excedido é sempre maior quando calculada pelo procedimento conservador.

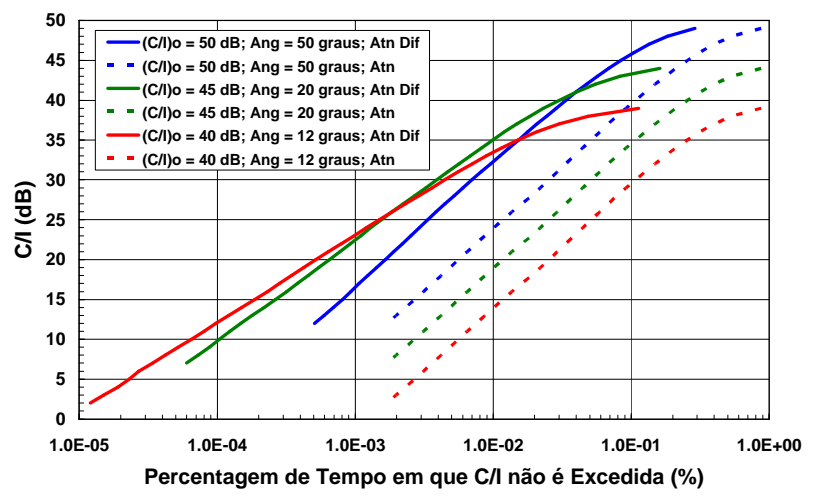

Figura 10. Distribuição cumulativa da relação C/I de acordo com os procedimentos conservador e realista, para três valores da relação $(\mathrm{C} / \mathrm{I})_{\mathrm{o}}$ ou, equivalentemente, da separação angular entre os enlaces 
Para avaliar o impacto dos procedimentos descritos acima na determinação do efeito da chuva sobre a indisponibilidade de um enlace e sobre a eficiência na utilização do espectro eletromagnético, será suposto que, desprezando-se os efeitos do ruído térmico, um enlace está indisponível quando $\mathrm{C} / \mathrm{I}=20 \mathrm{~dB}$. Como critério de qualidade para o dimensionamento do enlace, exige-se que, devido à chuva, a indisponibilidade ocorra, no máximo, durante $0,01 \%$ do tempo (isto é, durante 53 minutos do ano médio). Com base no procedimento conservador e considerando a possibilidade de outras interferências, seria necessário dimensionar o enlace com a margem de $50 \mathrm{~dB}$ e evitar a reutilização da mesma freqüência em enlaces com separações angulares inferiores a $50^{\circ}$. Portanto, apenas seis enlaces convergentes na estação central poderiam compartilhar a mesma freqüência na mesma polarização. Por outro lado, com base no procedimento realista e mesmo considerando a possibilidade de outras interferências, seria possível dimensionar o enlace com a margem de $40 \mathrm{~dB}$ e reutilizar a mesma frequiência em enlaces com separações angulares iguais a $12^{\circ}$. Portanto, cerca de trinta enlaces convergentes na estação central poderiam compartilhar a mesma frequiência na mesma polarização.

\section{CONCLUSÕES}

Apresentou-se um modelo não paramétrico para a determinação da distribuição cumulativa da atenuação diferencial devida à chuva entre dois enlaces convergentes operando em freqüências superiores a $10 \mathrm{GHz}$, baseado em extensão do modelo de Misme e Fimbel [8] original.

Em seguida, previões do modelo foram comparadas com resultados experimentais correspondentes, obtidos pelo CETUC/PUC-Rio em enlaces convergentes da Embratel situados na cidade de São Paulo [5],[7] e pelo Electrical Communication Laboratory no Japão [2], sendo apresentados argumentos capazes de explicar as diferenças observadas. Foram também apresentadas previsões do modelo para os efeitos das variações do ângulo entre os enlaces e dos seus comprimentos. Observou-se que, para ângulos inferiores a um determinado limiar, as distribuições cumulativas variavam rapidamente em função do incremento no ângulo entre os enlaces. Entretanto, a partir deste limiar, a mesma variação tornou-se extremamente lenta. Adicionalmente, o comprimento de um dos enlaces foi variado, sendo fixados tanto o comprimento do outro enlace quanto o ângulo entre eles. Observou-se que, para comprimentos inferiores a um segundo limiar, as distribuições cumulativas variavam em função do incremento no comprimento do enlace. Entretanto, a partir deste limiar, esta variação não mais ocorreu. Justificativas para estas observações serão apresentadas em artigo futuro.

Finalmente, foi avaliado o efeito da chuva sobre a indisponibilidade de um enlace e sobre a eficiência na utilização do espectro eletromagnético, com base em dois procedimentos para a determinação da distribuição cumulativa da relação $\mathrm{C} / \mathrm{I}(\mathrm{dB})$ entre as potências dos sinais desejado e interferente, supondo a existência de apenas dois enlaces convergentes. O primeiro procedimento de cálculo analisado é conservador e considera a atenuação $\mathrm{A}_{d}(\mathrm{~dB})$ devida à chuva no enlace desejado e despreza a atenuação $A_{i}(d B)$ no enlace interferente. $O$ segundo procedimento é realista e considera ambas as atenuações. Os resultados mostraram que o procedimento realista pode proporcionar dimensionamentos mais econômicos de enlaces e a utilização mais eficiente do espectro. Portanto, seria interessante examinar estes resultados mais detalhadamente e realizar medidas apropriadas, para também comprová-los experimentalmente.

\section{AGRADECIMENTOS}

As medidas realizadas pelo CETUC/PUC-Rio em enlaces da Embratel situados na cidade de São Paulo foram objeto do Aditivo 08 ao Convênio PUC-Rio/Embratel P-005/94. Os autores apresentam seus sinceros agradecimentos aos professionais da Embratel e aos colegas do CETUC/PUCRio envolvidos nos experimentos.

\section{REFERÊNCIAS}

[1] K. Morita, O. Sasaki, e A. Akeyama, "Differential rain attenuation on adjacent 20-GHz band links," IEEE Transactions on Antennas and Propagation, vol. 23, no. 2, pp. 300-301, Março 1975.

[2] K. Morita e I. Higuti, "Prediction of differential rain attenuation on adjacent microwave and millimeter wave links," Review of the Electrical Communication Laboratories, vol. 25. no. 1-2, pp. 96103, Janeiro-Fevereiro 1977.

[3] J. D. Kanellopoulus e S. G. Koukoulas, "Analysis of the rain outage performance of route diversity systems," Radio Science, vol. 22, no. 4, pp.549-565, Agosto, 1987.

[4] A. D. Panagopoulus e J. D. Kanellopoulus, "Statistics of differential rain attenuation on converging terrestrial propagation paths," IEEE Transactions on Antennas and Propagation, vol. 51, no, 9, Setembro 2003.

[5] L. A. R. Silva Mello, E. Costa, e R. S. L. Souza, "Rain attenuation measurements at 15 and $18 \mathrm{GHz}, "$ Electronics Letters, vol. 38, no. 4, pp. 197-198, Fevereiro 2002.

[6] K. S. Paulson, R. J. Watson, e I. S. Usman, "Diversity improvement estimation from rain radar databases using maximum likelihood estimation," IEEE Transactions on Antennas and Propagation, vol. 54, no. 1, pp. 168-174, Janeiro 2006.

[7] N. A. Perez Garcia, L. A. R Silva Mello, e M. S. Pontes, "Measurements and prediction of differential rain attenuation in convergent links," Electronics Letters, vol. 41, no. 17, pp. 942-943, Agosto 2005.

[8] P. Misme e J. Fimbel, "Détermination théorique et expérimentale de l'affaiblissement par la pluie sur um trajet radioélectrique," Annales desTélécommunications, vol. 30, no. 5-6, pp. 149-158, Novembro-Dezembro 1975.

[9] L. Stola, "An approach to compute the performance of route diversity systems at frequencies above $10 \mathrm{GHz}$," CSELT Rapporti Tecnici, vol. IX, no. 6, pp. 617-620, Dezembro 1981.

[10] Specific Attenuation Model for Rain for Use in Prediction Methods, ITU-R (2005), Recomendação ITU-R P.838-3.

[11] J. P. de Almeida e Albuquerque, J. M. P. Fortes, W. A. Finamore, Probabilidades, Variáveis Aleatórias e Processos Estocásticos. Rio deJaneiro: Interciência : PUC-Rio, 2008.

[12] J. O'Rourke, Computational Geometry in C. New York: Cambridge University Press, 2005. 